Pacific

Journal of

Mathematics

NONEXISTENCE OF LOCAL MINIMA OF SUPERSOLUTIONS FOR THE CIRCULAR CLAMPED PLATE

Hans-Christoph Grunau and Guido Sweers 


\title{
NONEXISTENCE OF LOCAL MINIMA OF SUPERSOLUTIONS FOR THE CIRCULAR CLAMPED PLATE
}

\author{
Hans-Christoph Grunau and Guido Sweers
}

\begin{abstract}
In general, superbiharmonic functions do not satisfy a minimum principle like superharmonic functions do, i.e., functions $u$ with $0 \not \equiv \Delta^{2} u \geq 0$ may have a strict local minimum in an interior point. We will prove, however, that when a superbiharmonic function is defined on a disk and additionally subject to Dirichlet boundary conditions, it cannot have interior local minima. For the linear model of the clamped plate this means that a circular plate, which is pushed from below, cannot bend downwards even locally.
\end{abstract}

The simple biharmonic function $u(x):=|x|^{2}$ shows that there are no classical local maximum/minimum principles for the biharmonic operator $\Delta^{2}$ (and for higher order elliptic operators at all). On the other hand it is known that boundary value problems like the clamped plate equation

$$
\left\{\begin{array}{l}
\Delta^{2} u=f \text { in } \Omega, \\
u\left|\partial \Omega=\frac{\partial u}{\partial \nu}\right| \partial \Omega=0,
\end{array}\right.
$$

enjoy some positivity properties. Here $\Omega \subset \mathbb{R}^{2}$ is a bounded smooth domain with exterior unit normal $\nu$ at $\partial \Omega$. This boundary value problem has an obvious physical interpretation. The solution $u$ gives the deflection of a plate of shape $\Omega$ from the equilibrium $u \equiv 0$, which is clamped horizontically and which is subject to the vertical force $f$. In this context there exist some positivity results: If the domain $\Omega$ is the unit disk $B=\left\{x \in \mathbb{R}^{2}:|x|<1\right\}$ (see $[\mathbf{B o}]$ ) or if $\Omega$ is close to the disk $B$ in a suitable sense (see [GS1]), then it is known that $0 \not \equiv f \geq 0$ implies $u>0$, i.e., upwards pushing yields (globally) upwards bending. So, at least in these domains, nonconstant supersolutions of the clamped plate equation (1) are strictly positive. Here we call a function $u \in C^{4}(\Omega) \cap C^{1}(\bar{\Omega})$ a supersolution of (1), if it solves (1) with some $f \geq 0$. It should be stressed that, in spite of the seemingly quadratic structure of (1), the so called Dirichlet boundary conditions (1.b) prevent us from iterating second order methods. 
Although existence/nonexistence of local minima is of no use for proving positivity of solutions, it would be interesting to have a more precise information about the shape of supersolutions to (1). So an interesting question to ask in this context would be:

May a solution $u$ to the clamped plate boundary value problem (1) have a local minimum in $\Omega$, although the right hand side is nonnegative: $f \geq 0$ and $f \not \equiv 0$ ?

There are (highly nontrivial) examples even for arbitrarily smooth convex domains $\Omega$ where the answer is affirmative, the first of which is due to Duffin $[\mathbf{D u}]$. Subsequently Garabedian could prove a striking result (see [Ga]): In a long thin ellipse $\Omega$ there exists a right hand side $0 \not \equiv f \geq 0$ such that the corresponding solution of (1) also has negative values and hence a (global and local) negative minimum in $\Omega$. For a more extensive survey on positivity results/nonpositivity examples we refer to [GS1].

In contrast to the examples above, we shall show that it is actually possible to exclude the existence of local minima, when $\Omega$ is a disk:

Theorem 1. Let $\Omega=B \subset \mathbb{R}^{2}$ be the unit disk. Assume that $u \in C^{4}(B) \cap$ $C^{1}(\bar{B})$ is a solution of the clamped plate equation (1) with some $0 \not \equiv f \geq 0$. Then $u$ has no local minimum in $B$.

We remark that this is not a one dimensional result, as neither $u$ nor $f$ are assumed to be radially symmetric. The radial analogue of Theorem 1 can be found in [So, Proposition 1], cf. also [Da, Theorem 2.4].

To prove the result we reduce our nonsymmetric problem to a radial one: A possible minimum will be moved to the origin by means of a suitable Moebius transform. After radialization one would obtain a radial nonconstant supersolution, which has an interior local minimum at the origin. Due to Soranzo's result just mentioned this is impossible.

We feel that positivity and absence of local minima should be related. Could one perhaps show that in those domains $\Omega$, where the Green function for the clamped plate equation (1) is positive, solutions with $0 \not \equiv f \geq 0$ are not only strictly positive, but don't have any local minimum in $\Omega$, too?

However, here we are restricted to the disk. Neither the proof of Theorem 1 below nor the proof of our positivity result for domains close to the disk in [GS1] seems to give any indication on how to treat such a conjecture.

We finish the introduction with a brief description of a further physical interpretation of Theorem 1 . Let the velocity field $\left(v_{1}, v_{2}\right)$ and the pressure $p$ be a solution of the linear Stokes system in $B \subset \mathbb{R}^{2}$ subject to zero boundary 
conditions and an exterior force field $\left(F_{1}, F_{2}\right)$ :

$$
\begin{cases}-\Delta v_{j}+p_{x_{j}}=F_{j} & \text { in } B, j=1,2, \\ v_{1, x_{1}}+v_{2, x_{2}}=0 & \text { in } B, \\ v_{j} \mid \partial B=0 & j=1,2 .\end{cases}
$$

Then for the stream funtion $u: \bar{B} \rightarrow \mathbb{R}, \quad u_{x_{1}}=-v_{2}, u_{x_{2}}=v_{1}$, normalized by the condition $u \mid \partial B=0$, Theorem 1 yields: If the vorticity $F_{2, x_{1}}-F_{1, x_{2}}$ of the force field is nonnegative (and not identically zero), then the stream function cannot have a local minimum in $B$. That means that around an interior rest point of the velocity field the fluid cannot rotate clockwise provided the vorticity of the force field is nonnegative. According to the above mentioned example of Garabedian $[\mathbf{G a}]$ this could actually happen e.g., in a long thin ellipse.

Moebius transforms. In what follows we will identify $\mathbb{R}^{2}$ and $\mathbb{C}$ and use real and complex notation simultaneously: $x=\left(x_{1}, x_{2}\right)=x_{1}+i x_{2}$. The dot "." denotes the multiplication in $\mathbb{C}: a \cdot x=a_{1} x_{1}-a_{2} x_{2}+i\left(a_{1} x_{2}+a_{2} x_{1}\right)$, while we use brackets for the scalar product in $\mathbb{R}^{2}:\langle a, x\rangle=a_{1} x_{1}+a_{2} x_{2}$. In case of holomorphic mappings $h$ we denote the complex derivative by $h^{\prime}$.

For $a \in B$ we consider the biholomorphic Moebius transform

$$
h: \bar{B} \rightarrow \bar{B}, \quad h(x)=\frac{a-x}{1-\bar{a} \cdot x}
$$

and its inverse $h^{-1}=h$. We have $h(B)=B, h(\partial B)=\partial B, h(0)=a$, $h(a)=0$. We know from Loewner [Loe] that Moebius transforms as in (3) and suitable simultaneous transformations of the dependent variable $u$ leave the biharmonic equation invariant. As we are interested in biharmonic inequalities we need a slightly more precise information:

Lemma 1. Let $u \in C^{4}(B, \mathbb{R})$. For some $a \in B$ we consider the Moebius transform $h$ from (3). For the $C^{4}$-function $v$ defined by

$$
v(x):=\frac{1}{\left|h^{\prime}(x)\right|} u(h(x)), \quad x \in B,
$$

we have

$$
\Delta^{2} v(x)=\left|h^{\prime}(x)\right|^{3}\left(\Delta^{2} u\right)(h(x)), \quad x \in B .
$$

Proof. Instead of the real variables $x_{1}, x_{2}$, we use the complex variables $x, \bar{x}$ and also $z=h(x), \bar{z}=\overline{h(x)}=\frac{\bar{a}-\bar{x}}{1-a \cdot \bar{x}}$.

One has $\frac{\partial}{\partial \bar{x}} h(x)=0, \frac{\partial}{\partial x} \overline{h(x)}=0, h^{\prime}=\frac{\partial}{\partial x} h(x)=\frac{|a|^{2}-1}{(1-\bar{a} \cdot x)^{2}}$ and

$$
h^{\prime \prime}(x)=\frac{|a|^{2}-1}{(1-\bar{a} \cdot x)^{3}} 2 \bar{a}=\frac{2 \bar{a}}{1-\bar{a} \cdot x} h^{\prime}(x) .
$$


In complex notation we have

$$
\left(1-|a|^{2}\right) v(x, \bar{x})=(1-\bar{a} \cdot x)(1-a \cdot \bar{x}) u(h(x), \overline{h(x)}) .
$$

Then

$$
\begin{aligned}
& \left(1-|a|^{2}\right) v_{x x}(x, \bar{x}) \\
& =-2 \bar{a}(1-a \cdot \bar{x}) h^{\prime} u_{z}+(1-\bar{a} \cdot x)(1-a \cdot \bar{x})\left(\left(h^{\prime}\right)^{2} u_{z z}+h^{\prime \prime} u_{z}\right) \\
& =(1-\bar{a} \cdot x)(1-a \cdot \bar{x})\left(h^{\prime}\right)^{2} u_{z z}
\end{aligned}
$$

and similarly

$$
\begin{aligned}
\left(1-|a|^{2}\right) v_{x x \bar{x} \bar{x}}(x, \bar{x}) & =(1-\bar{a} \cdot x)(1-a \cdot \bar{x})\left(h^{\prime}\right)^{2}\left(\overline{h^{\prime}}\right)^{2} u_{z z \bar{z} \bar{z}} \\
& =\left(1-|a|^{2}\right)\left|h^{\prime}\right|^{3} u_{z z \bar{z} \bar{z}}
\end{aligned}
$$

and the relation (5) follows by $\Delta^{2} u=16 u_{z z \bar{z} \bar{z}}$.

\section{Proof of Theorem 1.}

Let $u \in C^{4}(B) \cap C^{1}(\bar{B})$ be a solution of the clamped plate equation (1) with $\Omega=B$ and $0 \not \equiv f \geq 0$. We assume by contradiction that $u$ has a local minimum at $a \in B$.

From $u$ we want to construct a radial superbiharmonic function with homogeneous Dirichlet boundary conditions, which would also have a local minimum. According to [So] that will be impossible.

Before we may radialize the solution, we will move the point $a$, where $u$ has a local minimum, into the origin. For this purpose we consider the Moebius transform (3) and define $v \in C^{4}(B) \cap C^{1}(\bar{B})$ according to (4):

$$
v(x):=\frac{1}{\left|h^{\prime}(x)\right|} u(h(x)), \quad x \in \bar{B} .
$$

By means of (5) from Lemma 1 we see that $v$ solves a related clamped plate equation

$$
\left\{\begin{array}{l}
\Delta^{2} v=\left|h^{\prime}\right|^{3}(f \circ h) \text { in } B, \\
v\left|\partial B=\frac{\partial v}{\partial \nu}\right| \partial B=0 .
\end{array}\right.
$$

Now we radialize. As radialization and the Laplace operator commute, we see that the radially symmetric function

$$
w(x):=\frac{1}{2 \pi} \int_{|\xi|=1} v(|x| \xi) d \omega(\xi)
$$

is also in $C^{4}(B) \cap C^{1}(\bar{B})$ and solves the Dirichlet problem

$$
\left\{\begin{array}{l}
\Delta^{2} w=g \text { in } B \\
w\left|\partial B=\frac{\partial w}{\partial \nu}\right| \partial B=0 .
\end{array}\right.
$$


Here we have set

$$
g(x):=\frac{1}{2 \pi} \int_{|\xi|=1}\left(\left|h^{\prime}\right|^{3}(f \circ h)\right)(|x| \xi) d \omega(\xi) .
$$

Obviously we have $0 \not \equiv g \geq 0$.

Due to the dilation factor $\frac{1}{\left|h^{\prime}\right|}$ in the definition (4) of $v$ it is not clear whether or not $v$ has a local minimum in 0 . But for the radialization $w$ this is indeed the case. Using that $u$ has a local minimum in $a$, we conclude for $|x|$ small enough:

$$
\begin{aligned}
w(x) & =\frac{1}{2 \pi} \int_{|\xi|=1} \frac{1}{\left|h^{\prime}(|x| \xi)\right|} u(h(|x| \xi)) d \omega(\xi) \\
& \geq u(a)\left\{\frac{1+|a|^{2}|x|^{2}}{1-|a|^{2}}-\frac{|x|}{\pi\left(1-|a|^{2}\right)} \int_{|\xi|=1}\langle a, \xi\rangle d \omega(\xi)\right\} \\
& =\frac{1+|a|^{2}|x|^{2}}{1-|a|^{2}} u(a) \geq \frac{1}{1-|a|^{2}} u(a)=\frac{1}{\left|h^{\prime}(0)\right|} u(h(0))=w(0) .
\end{aligned}
$$

Here we used that $u(a) \geq 0$, which follows from $f \geq 0$ and from the positivity of the corresponding Green's function in $B$, see [Bo].

Let us sum up what we have shown. From our assumption that $u$ has a local minimum in some point $a \in B$ we could conclude that there is a radial supersolution $w$ of the clamped plate equation (7) with $0 \not \equiv g \geq 0$, which has a local minimum in 0 . (If $a \neq 0$, this minimum would be even strict.) We obtain a contradiction by a result of Soranzo [So, Proposition 1] (cf. also [Da, Theorem 2.4]), according to which $w$ is strictly radially decreasing in $|x| \in(0,1)$.

Remark. The same method applies to solutions of the clamped plate equation

$$
\left\{\begin{array}{l}
\Delta^{2} u=f \text { in } B \subset \mathbb{R}^{2}, \\
u\left|\partial B=0, \quad\left(-\frac{\partial u}{\partial \nu}\right)\right| \partial B=\varphi,
\end{array}\right.
$$

where the boundary datum $\varphi$, as well as the right hand side $f$, is assumed to be nonnegative (and one of these two not identically zero). It may seem unsatisfactory that the solution itself has to be prescribed homogeneously on $\partial B$, but also in [GS2] this boundary datum played a special role. There we were concerned with a perturbation theory for positivity in generalized clamped plate equations under inhomogeneous Dirichlet boundary conditions.

Acknowledgment. We are grateful to the referee for his very helpful comments on a first draft of this paper. 


\section{References}

[Bo] T. Boggio, Sulle funzioni di Green d'ordine m, Rend. Circ. Mat. Palermo, 20 (1905), 97-135.

[Da] R. Dalmasso, A priori estimates for some semilinear elliptic equations of order $2 m$, Nonlinear Anal., 29 (1997), 1433-1452.

[Du] R.J. Duffin, On a question of Hadamard concerning super-biharmonic functions, J. Math. Phys., 27 (1949), 253-258.

[Ga] P.R. Garabedian, A partial differential equation arising in conformal mapping, Pacific J. Math., 1 (1951), 485-524.

[GS1] H.-Ch. Grunau and G. Sweers, Positivity for perturbations of polyharmonic operators with Dirichlet boundary conditions in two dimensions, Math. Nachr., 179 (1996), 89-102.

[GS2] _ The role of positive boundary data in generalized clamped plate equations, Z. angew. Math. Phys. ZAMP, 49 (1998), 420-435.

[Loe] Ch. Loewner, On generation of solutions of the biharmonic equation in the plane by conformal mappings, Pacific J. Math., 3 (1953), 417-436.

[So] R. Soranzo, A priori estimates and existence of positive solutions of a superlinear polyharmonic equation, Dyn. Syst. Appl., 3 (1994), 465-487.

Received August 5, 1999.

FACHGRUPpe Mathematik

UNIVERSITÄT BAYREUTH

D-95440 BAYREUTH

Germany

E-mail address: Hans-Christoph.Grunau@uni-bayreuth.de

UNIVERSITY OF UTRECHT

NL-3508 TA UTRECHT

The Netherlands

Afdeling Toegepaste Wiskundige Analyse - Faculteit ITS

TU DELFT

MEKELWEG 4

NL-2628 CD DELFT

The Netherlands

E-mail address: G.H.Sweers@its.tudelft.nl 